\title{
Angiolipoma in a Dog
}

\author{
Mariana Correia Oliveira', Marcelo Jorge Chipitelli de Carvalho², Jade Manhães de Souza Bastos', \\ Isabella Jenifer Viana Soares', Gabriela de Carvalho Cid', Juliana Gomes Oliveira², \\ Ana Paula de Castro Pires ${ }^{3}$ \& Marilene de Farias Brito ${ }^{1}$
}

\begin{abstract}
Background: Angiolipoma is a benign tumor composed of endothelial cells and mature adipocytes. Tumors reported in domestic species include two variants; infiltrative or non-infiltrative. Bitches and intact males seem predisposed. This mesenchyme tumor is commonly mistaken with lipoma due to its soft texture and subcutaneous site and often requires histopathology to confirm its diagnosis. Microscopic examination also enables the evaluation of surgical margins and rule out possible infiltrative sites. Complete surgical excision is usually curative. This study reports a case of non-infiltrating angiolipoma in a dog.

Case: A 14-year-old mixed-breed dog was presented to a veterinary clinic in the city of Rio de Janeiro, RJ, Brazil. On palpation, a painless mass was noted, with high mobility and covered by intact hirsute skin in the right subcutaneous ventrolateral region. Computed tomography of the chest showed an expansive mass of uptake only from the edges of the soft tissues of the right subcutaneous ventrolateral region. The mass was homogeneous and well delimited, suggesting a neoplastic process. Subsequently, the mass was surgically removed, fixed in $10 \%$ buffered formalin, and sent for histopathological analysis. On macroscopic examination, the mass was well delimited, without skin coverage, and measured $2.3 \times 1.9 \times 0.6 \mathrm{~cm}$. The consistency was smooth and unctuous in appearance with a compact cream-colored surface with blackish multifocal spots. Under microscopy, the histological sections showed neoplasm of mature adipocytes and of endothelial cells of blood vessels benign were filled with a marked amount of red blood cells. Multifocal fibrin thrombi and a mild inflammatory infiltrate composed of lymphocytes and rare mast cells were evident. There was no infiltration in the regional skeletal musculature. Thus, a diagnosis of non-infiltrative angiolipoma was established.

Discussion: The diagnosis of non-infiltrating angiolipoma in this case was established through the results of histopathological examination. The occurrence of this neoplasm in dogs is uncommon, and the data reported in the veterinary medicine literature are scarce. However, in this study, it was found that the neoplasm on screening presented a behavior like that of lipomas, with noninvasive growth and the absence of local recurrence. The canine species does not commonly convey pain on palpation during a clinical examination, as observed in the present case. In humans, multiple angiolipoma nodules are common; this clinical presentation differs from that in animals, in which solitary nodules are generally observed. In dogs, as in the present case, they seem to have a predilection for the trunk. In animals, the pathogenesis of angiolipomas is not established, but in humans, it is based on theories that include the reaction to harmful stimuli and congenital malformation of adipose tissue. In humans, the presence of fibrin thrombi on the periphery of the region of cell proliferation are microscopic findings that can assist in the diagnosis of angiolipomas, an approach that was implemented in the present case. The occurrence of this neoplasm in dogs is uncommon, and the data reported in the veterinary medicine literature are scarce. The importance of an adequate description of angiolipomas is based on the need to provide information about its epidemiology, biological behavior, and prognosis.
\end{abstract}

Keywords: canine, hemangiolipoma, vascular neoplasia, lipoma, skin. 


\section{INTRODUCTION}

Angiolipomas are benign neoplasms of mesenchymal origin, characterized histologically by the proliferation of blood vessels and proliferation of adipocytes and can be classified as infiltrative or non-infiltrative [3]. In veterinary medicine, there are few reports of angiolipomas, and among all species, the most cited are canine [6,9]; however, although uncommon, the frequency of reports in humans is higher than that in animals [4]. Angiolipoma is found at subcutaneous sites, commonly in the trunk of young-adult and elderly animals, and exhibits macroscopic characteristics of an irregular, soft, encapsulated, and slightly blackened solitary mass $[6,10]$. The infiltrative variant, on the other hand, presents with rapid, invasive growth, and surgical excision with a margin is considered curative [7]. There are different methods that are effective as diagnostic aids, among which ultrasound and computed tomography are prominent $[5,6]$; however, histopathological examination helps elucidate the nature of the neoplasm, as well as evaluate the infiltration characteristics, exclude the differential diagnoses, and guide therapeutic approaches [3]. This article reports a case of non-infiltrating angiolipoma in a dog, in addition to describing the clinical and pathological characteristics of this neoplastic type.

\section{CASE}

A 14-year-old mixed-breed female dog was presented to a particular veterinary clinic in the city of Rio de Janeiro. On palpation, a painless mass was noted, with high mobility and covered by intact hirsute skin in the right subcutaneous ventrolateral region. In addition, computed tomography of the chest showed an expansive mass of uptake only from the edges of the soft tissues of the right subcutaneous ventrolateral region. The mass was homogeneous and well delimited, suggesting a neoplastic process. Subsequently, the mass was surgically removed, fixed in $10 \%$ buffered formalin, processed routinely, embedded in liquid paraffin, cut at 5 micrometer, and stained with Hematoxylin and Eosin ${ }^{1}$.

On macroscopic examination, the mass was well delimited, without skin coverage, and measured $2.3 \times 1.9 \times 0.6 \mathrm{~cm}$. The consistency was smooth and unctuous in appearance with a compact cream-colored surface with blackish multifocal spots. Under microscopy showed neoplasm of mature adipocytes and of endothelial cells of blood vessels benign were filled with a marked amount of red blood cells. The blood vessels were filled with a marked amount of red blood cells or slightly eosinophilic fluid (plasma). Multifocal fibrin thrombi and a mild inflammatory infiltrate composed of lymphocytes and rare mast cells were evident. There was no infiltration in the regional skeletal musculature. Thus, a diagnosis of non-infiltrative angiolipoma was established (Figures 1 and 2).

\section{DISCUSSION}

The diagnosis of non-infiltrating angiolipoma in this case was established through the results of histopathological examination, which was necessary to confirm the diagnosis and exclude other angiolipomatous neoplasms [7], as well as differentiate malignant variants, such as liposarcoma and hemangiosarcoma, which are important differential diagnoses [6]. Due to the scarcity

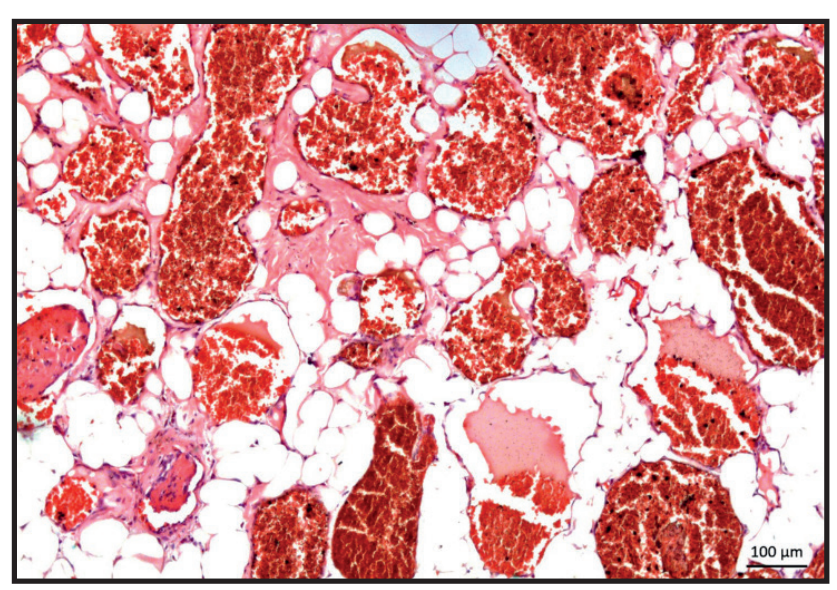

Figura 1. Photomicrograph of angiolipoma in a dog. Proliferation of benign mature adipocytes, pervading numerous blood vessels of different calibers [HE; Obj.20].

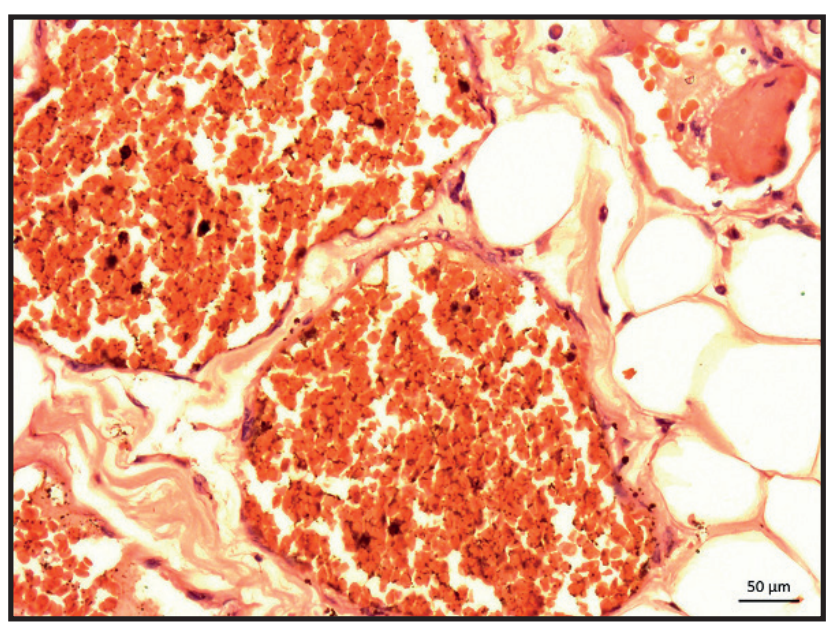

Figura 2. Photomicrograph of angiolipoma in a dog. Proliferation of blood vessels formed by endothelial cells with flattened and hyperchromatic nuclei, between the proliferation of mature adipocytes [HE; Obj.40]. 
of studies in veterinary medicine, especially of the non-infiltrative variant, data regarding epidemiological and clinicopathological characteristics are limited [3]. However, in this study, it was found that the neoplasm on screening presented a behavior like that of lipomas, with noninvasive growth and the absence of local recurrence. In humans, angiolipomas preferentially affect the subcutaneous tissue of the trunk and extremities [4]. In dogs, as in the present case, they seem to have a predilection for the trunk [7]. The subject of this study was a 14-year-old mixed-breed female dog. Previous reports showed no breed or sex predilection for the development of angiolipomas in dogs, and although older dogs seem to be more susceptible to the neoplasm; younger dogs are also affected [5-7,9,10].

The canine species does not commonly convey pain on palpation during a clinical examination, as observed in the present case [6,7]. In animals, the pathogenesis of angiolipomas is not established, but in humans, it is based on theories that include the reaction to harmful stimuli and congenital malformation of adipose tissue [1]. In humans, multiple angiolipoma nodules are common [8]; this clinical presentation differs from that in animals, in which solitary nodules are generally observed [6]. In humans, the presence of fibrin thrombi on the periphery of the region of cell proliferation are microscopic findings that can assist in the diagnosis of angiolipomas [2], an approach that was implemented in the present case and in other dogs [5-7].

In the present case, histopathology allowed for the diagnosis of non-infiltrating angiolipoma in a dog. The occurrence of this neoplasm in dogs is uncommon, and the data reported in the veterinary medicine literature are scarce. It is not known whether the lack of information is due to its rarity and/or diagnostic underreporting. Thus, the importance of an adequate description of angiolipomas is based on the need to provide information about its epidemiology, associated clinical signs, biological behavior, and prognosis.

\section{MANUFACTURER}

${ }^{1}$ EasyPath. Indaiatuba, SP, Brazil.

Declaration of interest. The authors report no conflicts of interest. The authors alone are responsible for the content and writing of the paper.

\section{REFERENCES}

1 Apostolakis S., Karagianni A., Mitropoulos A., Mantas C., Mavridis L., Filias P., Eftychiadis C. \& Vlachos K. 2020. Spinal angiolipoma: Presentation of two cases and review of the literature for the years 2012-2017. Neurocirugía. 31: 76-86. DOI: doi.org/10.1016/j.neucir.2019.04.005

2 Goldblum J., Weiss S. \& Folpe A.L. 2013. Benign lipomatous tumors. In: Enzinger F.M. \& Weiss S.W. (Eds). Soft Tissue Tumours. 6th edn. Philadelphia: Elsevier Saunders, pp.449-452.

3 Gross T.L., Ihrke P.J., Walder E.J. \& Affolter V.K. 2009. Tumores lipocíticos. In: Gross T.L., Ihrke P.J., Walder E.J. \& Affolter V.K. (Eds). Doenças de Pele do Cão e do Gato: Diagnóstico Clínico e Histopatológico. 2.ed. São Paulo: Roca, pp.750-761.

4 Hamano A., Suzuki K., Saito T., Kuwatsuru R., Oh S. \& Suzuki K. 2013. Infiltrating angiolipoma of the thoracic wall: a case report. Open Journal of Clinical Diagnostics. 3:19-22. DOI: 10.4236/ojcd.2013.32005

5 Kitshoff A.M., MIllward I.R., Williams J.H., Cliftb S.J. \& Kirbergerc R.M. 2010. Infiltrative angiolipoma of the parotid salivary gland in a dog. Journal of the South African Veterinary Association. 81:258-261. DOI: 10.4102/jsava. v81i4.158

6 Kraun M.B., Nelson N.C. \& Hollinger C. 2015. Imaging diagnosis-computed tomographic, surgical, and histopathologic characteristics of an infiltrative angiolipoma in a dog. Veterinary Radiology Ultrasound. 56: 31-35. DOI: 10.1111/ vru. 12178

7 Liggett A.D., Frazier K.S. \& Styer E.L. 2002. Angiolipomatous tumors in dogs and a cat. Veterinary Pathology. 39 : 286-289. DOI: doi.org/10.1354/vp.39-2-286

8 Lou X.H., Chen W.G., Ning L.G., Chen H.T. \& Xu G.Q. 2019. Multiple gastric angiolipomas: A case report. World Journal of Clinical Cases. 7: 778-784. DOI: 10.12998/wjcc.v7.i6.778

9 Reif U., Lowrie C.T. \& Fitzgerald S.D. 1998. Extradural spinal angiolipoma associated with bone lysis in a dog. Journal of the American Animal Hospital Association. 34: 373-376. DOI: 10.5326/15473317-34-5-373

10 Swamy M., Chandrapuria V.P. \& Kumar K. 2004. Angiolipoma in a dog. Indian Journal of Veterinary Pathology. 28: 141. DOI: 10.1354/vp.39-2-286 